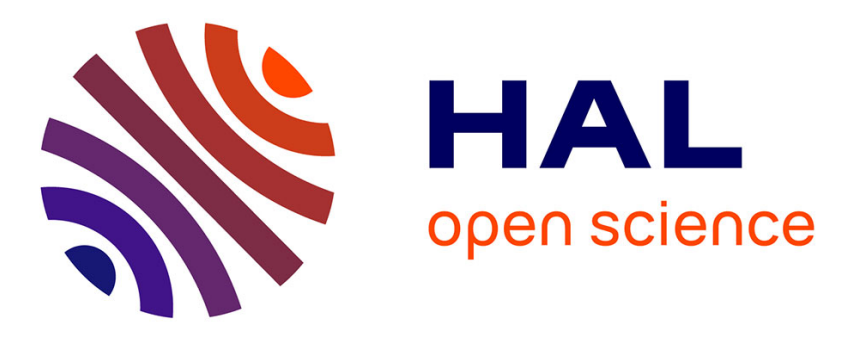

\title{
Biomechanical analysis of segmental lumbar lordosis and risk of cage subsidence with different cage heights and alternative placements in transforaminal lumbar interbody fusion
}

Sajjad Rastegar, Pierre-Jean Arnoux, Xiaoyu Wang, Carl-Éric Aubin

\section{To cite this version:}

Sajjad Rastegar, Pierre-Jean Arnoux, Xiaoyu Wang, Carl-Éric Aubin. Biomechanical analysis of segmental lumbar lordosis and risk of cage subsidence with different cage heights and alternative placements in transforaminal lumbar interbody fusion. Computer Methods in Biomechanics and Biomedical Engineering, 2020, 23 (9), pp.456-466. 10.1080/10255842.2020.1737027 . hal-03242397

\section{HAL Id: hal-03242397 \\ https://hal-amu.archives-ouvertes.fr/hal-03242397}

Submitted on 31 May 2021

HAL is a multi-disciplinary open access archive for the deposit and dissemination of scientific research documents, whether they are published or not. The documents may come from teaching and research institutions in France or abroad, or from public or private research centers.
L'archive ouverte pluridisciplinaire HAL, est destinée au dépôt et à la diffusion de documents scientifiques de niveau recherche, publiés ou non, émanant des établissements d'enseignement et de recherche français ou étrangers, des laboratoires publics ou privés.

\section{(이)( $)$}

Distributed under a Creative Commons Attribution - NonCommercial - NoDerivatives| 4.0 


\section{Biomechanical analysis of segmental lumbar lordosis and risk of cage}

2 subsidence with different cage heights and alternative placements in

3 transforaminal lumbar interbody fusion

4 Sajjad Rastegar ${ }^{\text {a,b,c }}$, Pierre-Jean Arnoux Ph.D. ${ }^{\text {c,d }}$, Xiaoyu Wang Ph.D. ${ }^{\text {a,b,c }}$,

5 Carl-Éric Aubin Ph.D., P.Eng. ${ }^{\text {a,b,c* } *}$

$6 \quad{ }^{a}$ Department of Mechanical Engineering, Polytechnique Montréal, Canada, ${ }^{b}$ Sainte-Justine University Hospital Center, Montreal, Canada, ${ }^{c}$ iLab Spine - International Laboratory Spine Imaging and Biomechanics, Canada/France; ${ }^{d}$ Laboratoire de Biomécanique Appliquée, UMRT24 IFSTTAR/Aix-Marseille Université, Marseille, France

11 Corresponding author:

12 Carl-Éric Aubin, Ph.D., P.Eng.

13 Full Professor

14 NSERC/Medtronic Industrial Research Chair in Spine Biomechanics

15 Polytechnique Montreal, Department of Mechanical Engineering

16 P.O. Box 6079, Downtown Station, Montreal (Quebec), H3C 3A7 CANADA

17 E-mail: carl-eric.aubin@polymtl.ca

18 Phone: 1 (514) 340-4711 ext. 2836; Fax: 1 (514) 340-5867

19 


\section{Biomechanical analysis of segmental lumbar lordosis and risk of cage \\ 2 subsidence with different cage heights and alternative placements in \\ 3 transforaminal lumbar interbody fusion}

5 Abstract

Cage subsidence in transforaminal lumbar interbody fusion (TLIF) is one of the concerns. The objective was to numerically assess the resulting segmental lumbar lordosis (SLL) and stresses at the bone-cage interface as functions of cage height (8- vs. 10-mm) and cage placement (oblique asymmetric, vs. anterior symmetric) for normal and. osteoporotic bone quality. A L4-L5 detailed finite element model of TLIF.was subjected to the functional loadings of $10 \mathrm{Nm}$ in the physiological planes after the application of a $400 \mathrm{~N}$ follower-load. The SLL was increased by $0.9^{\circ}(11 \%)$ and $1.0^{\circ}(13 \%)$, respectively in oblique asymmetric and anterior symmetric cage placement with $8-\mathrm{mm}$ height; they were $1.4^{\circ}(18 \%)$ and $1.7^{\circ}(21 \%)$ for the 10-mm cage. The maximum stresses at the cage-bone interface, in normal bone model, were increased up to $16 \%$ and $41 \%$ with the $10-\mathrm{mm}$ cage and asymmetric oblique placement, respectively, and they increased up to $16 \%$ and $43 \%$ in osteoporotic bone model. The greater cage resulted to a higher simulated SLL. Oblique asymmetric placement and the use of a greater cage may increase the risk of cage subsidence. Due to the lower mechanical strength of osteoporotic bone, the risk of cage subsidence should be higher.

Keywords: finite element analysis, TLIF (Transforaminal lumbar interbody fusion), cage subsidence, interbody cage, biomechanics, spine

Abstract word count: 196

Main-text word count: 4697

Number of tables: 3

Number of figures: 8 


\section{Introduction}

2 Transforaminal lumbar interbody fusion (TLIF) is a surgical procedure to restore the

3 intervertebral body height, the lumbar lordosis (LL), and spinal stability. This involves

4 the removal of the nucleus pulposus (NP) and a portion of the annulus fibrosus (AF),

5 followed by decompression of the segment and the placement of an interbody cage

6 through a unilateral approach. This is aimed at achieving an anterior interbody fusion in

7 addition to the posterior one by a solid segmental fixation (Agrawal and Resnick 2012;

8 Gum et al. 2016).

9 One of the mechanical complications of the TLIF surgical intervention is cage

10 subsidence, a situation where a cage enters into the vertebral body and consequently

11 results in the loss of intervertebral body height and segmental lumbar lordosis (SLL)

12 created intraoperatively by the instrumentation. Cage subsidence rates associated with

13 different cage designs and surgical techniques were reportedly from $10 \%$ to $38 \%$ (Le et

14 al. 2012; Malham et al. 2015; Lee et al. 2017). A cadaveric experimental study reported

15 that the subsidence stiffness and subsidence force with TLIF were significantly lower

$16(\mathrm{p}<0.01)$ than those for anterior (ALIF) and lateral (LLIF) lumbar interbody fusion

17 (Palepu et al. 2019). Several risk factors of cage subsidence in TLIF have been

18 identified, such as cage geometry (shapes and sizes) (Cho et al. 2008; Le et al. 2012;

19 Agarwal et al. 2013; Faizan et al. 2014; Kim JT et al. 2015; Deng et al. 2016; Kim CW

20 et al. 2016), single cage vs. paired cages (Xu et al. 2013), use of unilateral posterior

21 fixation vs. bilateral one (Chen et al. 2012; Ambati et al. 2015), and trabecular bone

22 volume fraction (Palepu et al. 2019).

23 Biomechanical analysis using finite element models showed that a larger cage

24 footprint (e.g., 490 vs. $280 \mathrm{~mm} 2$ ) allowed to bear about $300 \%$ more functional load and

25 reduced the maximum stresses by about 50\%, resulting in a lower risk of cage 
1 subsidence (Faizan et al. 2014). Biconvex shapes were shown to allow better cage

2 fitting, but with loads more concentrated in the medial region of the endplates with

3 relatively lower mechanical strength than peripheral cortical bone, thus higher risk of

4 cage subsidence (Cho et al. 2008). Using paired- vs. single-cage configurations resulted

5 in $55.2 \%$ lower stress at the bone-cage interface (49.77 vs. $77.23 \mathrm{MPa}$ ) and

6 subsequently lower risk of cage subsidence (Xu et al. 2013).

7 A thicker cage is generally more effective for SLL restoration, but requires more

8 intervertebral distraction for its placement, which increases the risk of cage subsidence

9 due to the higher compressive forces at the bone-cage interface ( $8.8 \mathrm{~N}$ with 6-mm cage

10 vs. $21.5 \mathrm{~N}$ with 8 -mm cage in a biomechanical experiments using cadaveric lumbar

11 spines) (Truumees et al. 2008; Le et al. 2012). Clinical studies showed that a kidney-

12 shape cage placed $16 \%$ more anteriorly vs. a medial placement of a bullet-shape cage,

13 resulted in an SSL increase of $2.11^{\circ}$ (Kim JT et al. 2015) and reduced the risk of cage

14 subsidence by shifting the bone-cage contact more to the peripheral region of the

15 endplates with superior mechanical strength. The results of a controlled cadaveric test

16 reported that using shorter cage (with length of 22- vs. 27-mm) in TLIF can potentially

17 restore the segmental lordosis up to $8.7^{\circ}$ (Robertson et al. 2018). Wedged cages (vs. flat

18 cages) are reported to allow better lordosis restoration; increasing the wedge angle from

$194^{\circ}$ to $15^{\circ}$, and increased the resulting SLL from $2.6^{\circ}$ to $6.5^{\circ}$ (Hong et al. 2017).

20 Clinical studies, experiments using cadaveric spines, and numerical analyses

21 have been done on the use of interbody cages of different shapes, configurations, and

22 heights. However, the effects of essential cage parameters are not yet fully understood;

23 therefore, systematic biomechanical investigations remain to be performed to acquire

24 comprehensive biomechanical facts to help realize and reduce the risk of cage

25 subsidence. The objective of this study was to numerically assess the biomechanics of 
1 TLIF in terms of the resulting SLL and stresses at the bone-cage interface as functions

2 of the cage height and its placement strategy with two tested bone qualities. This

3 objective aims at comparing two common cage placement strategy in surgical procedure

4 while surgeon may choose between two cages with different heights.

\section{$6 \quad$ Material and methods}

\section{$7 \quad$ Finite element model of the L4-L5 segment}

8 A detailed finite element model (FEM) of L4-L5 functional spinal unit was created

9 based on a previously developed and validated FEM of the spine (El-Rich et al. 2008;

10 El-Rich et al. 2009) (Figure 1a). The FEM was adapted and refined to simulate the

11 biomechanics of the TLIF, including intervertebral space preparation, cage insertion,

12 and posterior fixation (Agrawal and Resnick 2012; Gum et al. 2016). The geometric

13 model of the spine was reconstructed using medical images acquired through a CT-scan

14 (0.6 mm slice thickness) of a $50^{\text {th }}$ percentile healthy man (El-Rich et al. 2008; El-Rich

15 et al. 2009). The model consisted of the vertebral body (cancellous and cortical bones),

16 posterior arches, intervertebral disc, facet joints, and seven ligaments, i.e. the anterior

17 longitudinal ligament (ALL), posterior longitudinal ligament (PLL), ligamentum flavum

18 (LF), capsular ligaments (CL), intertransverse ligament (ITL), interspinous ligament

19 (ISL), and supraspinous ligament (SSL) (Figure 1a).

20 Each vertebra was meshed with 4-node solid elements, representing the

21 trabecular bone enveloped by a layer of cortical bone with changing thickness in five

22 regions: endplates and anterior walls of the vertebral body $(0.4 \mathrm{~mm})$, upper pedicle (2

$23 \mathrm{~mm})$, lower pedicle $(1.87 \mathrm{~mm})$, posterior processes $(1 \mathrm{~mm})$, and insertion area of

24 pedicle screws (0.8 mm) (Silva et al. 1994; Hirano et al. 1997; Bianco et al. 2017) 
1 (Figure 1b). The AF was modelled with five concentric layers of 8-node solid elements

2 between the two vertebrae, reinforced by spring elements to simulate the collagen fibres

3 oriented at $\pm 35^{\circ}$. The NP was meshed with 8-node elements. All ligaments were meshed

4 with 4-node shell elements, except the CL, which was represented by 3-node shell

5 elements. To balance the computation cost and analysis accuracy for this study, we

6 performed a mesh convergence study to determine adequate element sizes (Figure 1a

7 and Figure 1b).

Non-linear material properties were implemented to model the mechanical

9 behaviour of the spinal elements in physiological loading conditions. The cortical and

10 trabecular bones were modelled as homogenous isotropic materials governed by the

11 elastoplastic Johnson-Cook constitutive law (Wagnac et al. 2012). The NP and AP were

12 modelled as Mooney-Rivlin hyperelastic material while collagen fibres were

13 incorporated as one-dimensional spring elements acting in tension only. The non-linear

14 behaviour of the spinal ligaments was modelled with a generalized Maxwell-Kelvin-

15 Voigt constitutive law, and the failure criteria was incorporated based on the maximum

16 tensile strain level (Wagnac et al. 2012). The material properties of the elements were

17 initially defined using numerical results from the literature (Tables 1, 2, and 3). Material

18 properties of osteoporotic bone were modelled by reducing Young's modulus of cortical

19 (33\%) and trabecular bone (66\%) (Polikeit et al. 2003; Salvatore et al. 2018). To model

20 the zygapophyseal facet joints, a general purpose contact was used with an initial gap of

$210.5 \mathrm{~mm}$ (Faizan et al. 2014) and Coulomb friction coefficient of 0.2 (Li et al. 2015)

22 between the two facets of the articulation. Tied contacts were modelled between the

23 ligaments and cortical bone at their attachment sites. The mechanical properties of the

24 aforementioned modelling elements were adjusted and calibrated such that the load-

25 displacement results of functional loading simulations corresponded to the results from 
1 experiments on cadaveric lumbar spines (Heuer et al. 2007). To validate the FEM, the

2 ROMs of the intact model under simulated pure bending of $8 \mathrm{Nm}$ in flexion-extension,

3 lateral bending, and axial rotation were compared with the reported ROMs of similar

4 experimental cadaveric tests (Dahl et al. 2013; Jaramillo et al. 2016). To support the

5 decisions in the context of use (COU) which was the risk of cage subsidence in TLIF,

6 the credibility of developed FEM was stablished. For this purpose, the assumption and

7 model inputs were tested in their ranges to assure that the results are still applicable to

8 the COU with the quantified uncertainty of the predictions.

\section{Simulation of TLIF procedure}

11 The surgical procedure of TLIF was modelled by partial discectomy through the

12 unilateral approach was modelled by removing the elements corresponding to the

13 posterior-left portion of the AF and NP. A facetectomy was simulated by removing the

14 elements corresponding to the zygapophyseal joint to virtually make a window for the

15 cage insertion (Figure 2Error! Reference source not found.a). Four pedicle screws (40

$16 \mathrm{~mm}$ long, $6.5 \mathrm{~mm}$ diameter; CD HORIZON® LEGACY ${ }^{\mathrm{TM}}$, Medtronic, Memphis TN)

17 were modelled as rigid bodies, and their external surfaces were meshed with triangular

18 shell elements. They were aligned with their corresponding vertebra based on a typical

19 lumbar pedicle screw insertion technique (Agarwal et al. 2013; Bianco et al. 2017). To

20 identify the proper element size, a mesh convergence study initially was conducted by

21 testing various element sizes (from 0.5 to $1.5 \mathrm{~mm}$ ) at the endplate-cage interface of the

22 oblique asymmetric placement of $10-\mathrm{mm}$ cage, up until the variation of the maximum

23 Von-Mises stress at the bone-cage interface was lower than 5\%. Boolean operations

24 between the screw and the vertebral models were performed to remove the cortical layer 
1 and trabecular core model elements (Bianco et al. 2017). A point-to-surface contact

2 with a Columb friction of 0.2 was modelled to represent the bone-screw interface.

4 interbody cage, Medtronic, Memphis TN). The length and width of the models were 26

$5 \mathrm{~mm}$ and $10 \mathrm{~mm}$, respectively. Two cage heights were tested, i.e. 8 and $10 \mathrm{~mm}$. For each

6 model, we tested the oblique asymmetric and anterior symmetric placements, a total of

7 four interbody cage scenarios (Figure 3). The cages were meshed with 4-node

8 tetrahedral elements of $1.0 \mathrm{~mm}$, and material properties of polyether-ether-ketone

9 (PEEK) were assigned ( $\mathrm{E}=3.4 \mathrm{GPa}$ and $v=0.4$ (Faizan et al. 2014)). The modelling of

10 the cage insertion was based on the documented surgical technique (Agarwal et al.

11 2013). First, the cage model was aligned to the superior endplate of L5, and a node-to-

12 surface contact with a minimum distance of $0.5 \mathrm{~mm}$ and Columb friction coefficient of

13 0.2 was applied to the interface. Then, a distractive force was applied between L4 and

14 L5 such that the intervertebral body space increased and there was no interference

15 between the cage and endplate geometries. Finally, the loads were released after node-

16 to-surface contact was modelled between the cage and the adjoining endplates (Figure

17 2b). The SSL was assessed before and after the simulation of the cage placement by

18 measuring the angle between the superior endplate of L4 and inferior endplate of L5

19 (Hong et al. 2017). After the simulation of the cage insertion, two titanium rod (4.5 mm)

20 models were aligned with screw head saddles and tied contacts were modelled between

21 them to simulate the posterior fixation (Figure 2c). The rods were meshed with 4-node

22 tetrahedral solid elements of $0.5 \mathrm{~mm}$ characteristic length, and the material properties of

23 Titanium alloy were adapted from literature $(\mathrm{E}=115 \mathrm{GPa}$ and $v=0.34$ (Faizan et al.

24 2014)) 
2 was modelled as a $400 \mathrm{~N}$ follower-load to the superior elements of L4 with the inferior

3 endplate of L5 fixed in space. A 10-Nm functional load was simulated in the three

4 anatomical planes, respectively, to simulate flexion (Fe), extension (Ex), right lateral

5 bending (RLB), left lateral bending (LLB), right axial rotation (RAR), and left axial

6 rotation (LAR). The ROM and maximum Von-Mises stresses were computed as a

7 measure of the risk of cage subsidence.

All the simulations were performed using the RADIOSS v14.0 finite element

9 package (Altair Engineering Inc., Troy, USA) in a quasi-static condition (Bianco et al. 10 2017).

\section{Results}

13 The resulting ROMs of the non-instrumented FEM of the L4-L5 segment were $9.3^{\circ}$,

$147.6^{\circ}$, and $4.1^{\circ}$ in flexion-extension, lateral bending, and axial rotation, respectively.

15 These results were within the range of reported ROM of experimental cadaveric studies

16 in the literature (Dahl et al. 2013; Jaramillo et al. 2016) (Figure 4). With the simulated

17 normal bone quality, the anterior symmetric and oblique asymmetric placement of the

18 cages increased the SLL by $0.9^{\circ}$ and $1.0^{\circ}$, respectively, for the $8-\mathrm{mm}$ height cage, and

19 by $1.4^{\circ}$ and $1.7^{\circ}$ for the $10-\mathrm{mm}$ one. SLL restorations with simulated osteoporosis were

20 within $1.2 \%$ to those with the normal bone quality. With normal bone, the simulated

21 ROMs of the FSU after the TLIF procedure were lower than $1^{\circ}$ in all loading directions,

22 while they ranged from $2^{\circ}$ to $8^{\circ}$ with the un-instrumented FSU (Figure $5 a$ ). With

23 simulated osteoporosis, the ROMs were slightly (about 8\%) higher than those with

24 normal bone (Figure 5b). Oblique asymmetric vs. anterior symmetric placement 
1 increased the ROM by $66 \%$ and $72 \%$ for the simulated normal and osteoporotic bone,

2 respectively. Insertion of the 8-mm cage vs. the $10-\mathrm{mm}$ one increased the ROM of the

3 instrumented segment up to $43 \%$ and $48 \%$ in simulated normal and osteoporotic bone

4 models, respectively.

For the 8-mm cage with normal bone quality, the maximum stresses at the bone-

6 cage interface ranged from 82.1 to $98.4 \mathrm{MPa}$ (anterior symmetric placement) and from

7117.9 to $155.5 \mathrm{MPa}$ (oblique asymmetric placement) (Figure 6a). For the 10-mm cage,

8 they were from 88.2 to $107.2 \mathrm{MPa}$ (anterior symmetric) and between 134.4 and 176.4

$9 \mathrm{MPa}$ (oblique asymmetric) (Figure 6a). With osteoporosis, stresses at the bone-cage

10 interface were about $2.5 \%$ lower (Figure $6 \mathrm{~b}$ ). Oblique asymmetric as compared to the

11 anterior symmetric cage placement increased the maximum stresses by up to $41 \%$ and

$1243 \%$ for the simulated normal and osteoporotic bone, respectively. Insertion of the 10-

$13 \mathrm{~mm}$ cage vs. the 8 -mm one increased the maximum stresses by up to $16 \%$ in simulated

14 normal and osteoporotic bone models. The stress on the superior endplate of L5 is

15 displayed on Figure 7 for the 4 tested configurations under simulated flexion moment of

$1610 \mathrm{~N} \mathrm{~m}$ and $400 \mathrm{~N}$ follower-load.

17 For the 8-mm cage with normal bone quality, the maximum stresses in the

18 posterior rods were between 128.9 and $230.3 \mathrm{MPa}$ (anterior symmetric) and between

19114.9 and 326.6 MPa (oblique asymmetric) (Figure 8a). For the 10-mm cage, they

20 ranged from 60.3 to $218.0 \mathrm{MPa}$ (anterior symmetric) and from 69.6 to $262.5 \mathrm{MPa}$

21 (oblique asymmetric) (Figure 6a). With osteoporosis, stresses in the posterior rods

22 increased up to about $120 \%$ (Figure 8b). Oblique asymmetric vs. anterior symmetric

23 placement increased the maximum stresses by up to $55 \%$ and $48 \%$ for the simulated

24 normal and osteoporotic bone, respectively. In simulations with oblique asymmetric

25 placement, stresses in the rod on the opposite side of the cage were higher than the other 
1 rod. Insertion of the 8-mm cage vs. the 10-mm one increased the maximum stresses up

2 to $59 \%$ and $54 \%$ in simulated normal and osteoporotic bone, respectively.

\section{Discussion}

5 A larger SLL restoration was observed in the simulations with a $10-\mathrm{mm}$ vs. 8 -mm cage.

6 This was expected from a geometric point of view because greater cage height means

7 greater anterior intervertebral distance, thus higher SLL. Consequently, stresses at the

8 bone-cage interface in simulations of $10-\mathrm{mm}$ cage were always higher than the $8-\mathrm{mm}$

9 cage. Cages of greater height required more intervertebral distraction for its proper

10 placement, which initiated a higher compression force at the bone-cage interface

11 generated by the tightening of the soft tissues, which translated in higher structural

12 stiffness and lower ROM due to the non-linear mechanical behaviour of the

13 intervertebral ligaments. This could explain why the maximum stresses in the rods with

14 the $10-\mathrm{mm}$ cage were lower than the $8-\mathrm{mm}$ cage. The stresses generated by the compression forces as a function of cage height agreed with the reported experiments with cadaveric lumbar spines (Truumees et al. 2008; Ambati et al. 2015).

The simulated SLL restoration with the anterior symmetric cage placement was very close to that with the oblique asymmetric placement, but the maximum stresses at the bone-cage interface with anterior symmetric placement were consistently lower than those with oblique asymmetric placement. This may be explained by the fact that the

21 resultant force at the bone-cage interface with anterior symmetric placement has a

22 longer lever arm with respect to the posterior fixation, providing a mechanical

23 advantage to balance the external loads. In this standpoint, with the use of similar

24 interbody cage footprints and in the presence of a smaller compression force at the bone-cage interface, lower stress is expected with the anterior cage placement. 
1 Compared with oblique asymmetrical placement, the anterior symmetrically placed

2 cage had more bone-cage contact area in the anterior part of the intervertebral body

3 space (Figure 4) where the endplates have superior mechanical strength (Tsitsopoulos et

4 al. 2012; Faizan et al. 2014). Increased stresses due to oblique asymmetric vs. anterior

5 symmetric (41\%) seems to have a significant effect on the stress distribution at the

6 bone-cage interface. This difference is clinically important because the maximum stress

7 at the interface, in some cases, exceeds the failure stress of cortical bone (126 MPa)

8 (Hansen et al. 2008). On the other hand, using 10-mm cage does not significantly affect

9 the risk of subsidence since the value of the maximum stresses are still below the failure

10 stress of cortical bone. With the oblique asymmetric placement, reaction forces from the

11 rods had, therefore, shorter lever arms with respect to the cage centre - fulcrum point

12 between the upper and lower vertebral bodies, resulting in a higher stress in the rods to

13 balance the loads.

14 There was no difference in SLL restoration between normal and osteoporosis

15 bones. Although the maximum stresses at the bone-cage interface for the simulated

16 osteoporosis were identical to those of modelled normal bone, the risk of cage

17 subsidence should be higher because the osteoporotic bones also have between $20 \%$ to

$1840 \%$ lower mechanical strengths due to decreased bone mineral density (Dickenson et

19 al. 1981; Bono and Einhorn 2003). Also, clinical studies showed that the risk of cage

20 subsidence in osteoporosis spines was about 3 times higher than in spines with normal

21 bone (Formby et al. 2016; Oh et al. 2017). The simulated osteoporosis bones had lower

22 stiffness and provided less support of the functional loads as compared to the normal

23 bones, making the rods subjected to higher loads and stresses.

24 Some simplifications and approximations were made in the modelling and

25 simulations in this study (i.e. the cortical and trabecular bones were modelled as 
1 homogenous isotropic materials, the geometry and mechanical properties of the FSU

2 were based on a generic $50^{\text {th }}$ model, and screw insertion was model as a geometric

3 Boolean operation between the screw and the vertebral models and with a contact

4 definition between the two). These modelling simplifications and approximations are

5 considered to have limited effects on the conclusions because this study focused on the

6 relative effects of the cage height, cage placement and bone quality which are common

7 in TLIF for most of the cases. The presented modelling technique might be adapted to

8 examine the biomechanics of multi-level TLIF, as well as the performance of any other

9 interbody cages in terms of risk of cage subsidence.

\section{Conclusion}

12 A detailed FEM was developed to simulate the biomechanics of the TLIF procedure.

13 The FEM allowed the assessment of the effects of the cage height, cage placement, and

14 bone quality on the SLL restoration and risk of the cage subsidence. It was found that

15 10- vs 8-mm cage height resulted in up to $0.7^{\circ}$ higher SLL restoration and $16 \%$ higher

16 stresses at the bone-cage interface. Oblique asymmetric placement vs. anterior

17 symmetric placement had almost similar SLL restoration, but the stresses at the bone-

18 cage interface were up to $43 \%$ higher. Bone quality did not affect the achieved SLL; a

19 higher risk of cage subsidence is expected for the osteoporotic spines although the

20 maximum stresses at the bone-cage interface were $2.5 \%$ lower. The FEM presented in

21 this study was shown to be a relevant tool to assess the biomechanics of TLIF. It could

22 be further adapted to further assess the biomechanics of any interbody cage design, as

23 well as to evaluate reported clinical findings towards the improvement of the TLIF

24 procedure. 


\section{Acknowledgement}

3 This study was financially supported by Medtronic and the Natural Sciences and

4 Engineering Research Council of Canada (Industrial Research Chair program with

5 Medtronic of Canada).

6 


\section{References}

Agarwal A, Palepu V, Agarwal AK, Goel VK, Yildirim ED. 2013. Biomechanical evaluation of an endplate-conformed polycaprolactone-hydroxyapatite intervertebral fusion graft and its comparison with a typical nonconformed cortical graft. Journal of biomechanical engineering. 135(6):61005-61009.

Agrawal BM, Resnick D. 2012. Transforaminal Lumbar Interbody Fusion. Schmidek and Sweet Operative Neurosurgical Techniques. Philadelphia: W.B. Saunders; p. 1951-1954.

Ambati DV, Wright EK, Jr., Lehman RA, Jr., Kang DG, Wagner SC, Dmitriev AE. 2015. Bilateral pedicle screw fixation provides superior biomechanical stability in transforaminal lumbar interbody fusion: a finite element study. The spine journal. 15(8):1812-1822.

Bianco RJ, Arnoux PJ, Wagnac E, Mac-Thiong JM, Aubin CE. 2017. Minimizing Pedicle Screw Pullout Risks: A Detailed Biomechanical Analysis of Screw Design and Placement. Clinical Spine Surgery. 30(3):E226-E232.

Bono CM, Einhorn TA. 2003. Overview of osteoporosis: pathophysiology and determinants of bone strength. European spine journal. 12(2):S90-96.

Chen SH, Lin SC, Tsai WC, Wang CW, Chao SH. 2012. Biomechanical comparison of unilateral and bilateral pedicle screws fixation for transforaminal lumbar interbody fusion after decompressive surgery--a finite element analysis. BMC musculoskeletal disorders. 13:72.

Cho W, Wu C, Mehbod AA, Transfeldt EE. 2008. Comparison of cage designs for transforaminal lumbar interbody fusion: a biomechanical study. Clinical biomechanics (Bristol, Avon). 23(8):979-985. eng.

Dahl MC, Ellingson AM, Mehta HP, Huelman JH, Nuckley DJ. 2013. The biomechanics of a multilevel lumbar spine hybrid using nucleus replacement in conjunction with fusion. The spine journal. 13(2):175-183. eng.

Deng QX, Ou YS, Zhu Y, Zhao ZH, Liu B, Huang Q, Du X, Jiang DM. 2016. Clinical outcomes of two types of cages used in transforaminal lumbar interbody fusion for the treatment of degenerative lumbar diseases: n-HA/PA66 cages versus PEEK cages. Journal of materials science Materials in medicine. 27(6):102.

Dickenson RP, Hutton WC, Stott JR. 1981. The mechanical properties of bone in osteoporosis. J Bone Joint Surg Br. 63-b(2):233-238. 
1 El-Rich M, Arnoux PJ, Wagnac E, Brunet C, Aubin CE. 2009. Finite element investigation of the loading rate effect on the spinal load-sharing changes under impact conditions. Journal of biomechanics. 42(9):1252-1262.

El-Rich M, Wagnac E, Arnoux PJ, Aubin CE. 2008. Detailed modeling of the lumbar spine for trauma applications: preliminary results. Comput Method Biomec. 11(sup001):93-94.

Faizan A, Kiapour A, Kiapour AM, Goel VK. 2014. Biomechanical analysis of various footprints of transforaminal lumbar interbody fusion devices. J Spinal Disord Tech. 27(4):E118-127.

Formby PM, Kang DG, Helgeson MD, Wagner SC. 2016. Clinical and Radiographic Outcomes of Transforaminal Lumbar Interbody Fusion in Patients with Osteoporosis. Global spine journal. 6(7):660-664.

Gum JL, Reddy D, Glassman S. 2016. Transforaminal Lumbar Interbody Fusion (TLIF). JBJS essential surgical techniques. 6(2):e22. eng.

Hansen U, Zioupos P, Simpson R, Currey JD, Hynd D. 2008. The Effect of Strain Rate on the Mechanical Properties of Human Cortical Bone. Journal of Biomechanical Engineering. 130(1).

Heuer F, Schmidt H, Klezl Z, Claes L, Wilke HJ. 2007. Stepwise reduction of functional spinal structures increase range of motion and change lordosis angle. Journal of biomechanics. 40(2):271-280.

Hirano T, Hasegawa K, Takahashi HE, Uchiyama S, Hara T, Washio T, Sugiura T, Yokaichiya M, Ikeda M. 1997. Structural characteristics of the pedicle and its role in screw stability. Spine. 22(21):2504-2509; discussion 2510.

Hong TH, Cho KJ, Kim YT, Park JW, Seo BH, Kim NC. 2017. Does Lordotic Angle of Cage Determine Lumbar Lordosis in Lumbar Interbody Fusion? Spine. 42(13):E775-E780.

Jaramillo HE, Puttlitz CM, McGilvray K, Garcia JJ. 2016. Characterization of the L4L5-S1 motion segment using the stepwise reduction method. Journal of biomechanics. 49(7):1248-1254.

Kim CW, Doerr TM, Luna IY, Joshua G, Shen SR, Fu X, Wu AM. 2016. Minimally Invasive Transforaminal Lumbar Interbody Fusion Using Expandable Technology: A Clinical and Radiographic Analysis of 50 Patients. World neurosurgery. 90:228-235. 
1 Kim JT, Shin MH, Lee HJ, Choi DY. 2015. Restoration of lumbopelvic sagittal alignment and its maintenance following transforaminal lumbar interbody fusion (TLIF): comparison between straight type versus curvilinear type cage. Eur Spine J. 24(11):2588-2596.

\section{Le TV, Baaj AA, Dakwar E, Burkett CJ, Murray G, Smith DA, Uribe JS. 2012.} Subsidence of polyetheretherketone intervertebral cages in minimally invasive lateral retroperitoneal transpsoas lumbar interbody fusion. Spine. 37(14):12681273.

Lee N, Kim KN, Yi S, Ha Y, Shin DA, Yoon DH, Kim KS. 2017. Comparison of Outcomes of Anterior, Posterior, and Transforaminal Lumbar Interbody Fusion Surgery at a Single Lumbar Level with Degenerative Spinal Disease. World neurosurgery. 101(Supplement C):216-226.

Li J, Shang J, Zhou Y, Li C, Liu H. 2015. Finite Element Analysis of a New Pedicle Screw-Plate System for Minimally Invasive Transforaminal Lumbar Interbody Fusion. PloS one. 10(12):e0144637.

Malham GM, Parker RM, Blecher CM, Seex KA. 2015. Assessment and classification of subsidence after lateral interbody fusion using serial computed tomography. Journal of Neurosurgery Spine. 23(5):589-597.

Oh KW, Lee JH, Lee JH, Lee DY, Shim HJ. 2017. The Correlation Between Cage Subsidence, Bone Mineral Density, and Clinical Results in Posterior Lumbar Interbody Fusion. Clinical Spine Surgery. 30(6):E683-E689.

Palepu V, Helgeson M, Molyneaux-Francis M, Nagaraja S. 2019. The effects of bone microstructure on subsidence risk for ALIF, LLIF, PLIF, and TLIF spine cages. Journal of Biomechanical Engineering. 141(3): 031002..

Polikeit A, Nolte LP, Ferguson SJ. 2003. The effect of cement augmentation on the load transfer in an osteoporotic functional spinal unit: finite-element analysis. Spine. 28(10):991-996.

Salvatore G, Berton A, Giambini H, Ciuffreda M, Florio P, Longo UG, Denaro V, Thoreson A, An KN. 2018. Biomechanical effects of metastasis in the osteoporotic lumbar spine: A Finite Element Analysis. BMC musculoskeletal disorders. 19(1):38. 
1 Silva MJ, Wang C, Keaveny TM, Hayes WC. 1994. Direct and computed tomography thickness measurements of the human, lumbar vertebral shell and endplate. Bone. 15(4):409-414.

Truumees E, Demetropoulos CK, Yang KH, Herkowitz HN. 2008. Effects of disc height and distractive forces on graft compression in an anterior cervical corpectomy model. Spine. 33(13):1438-1441.

Tsitsopoulos PP, Serhan H, Voronov LI, Carandang G, Havey RM, Ghanayem AJ, Patwardhan AG. 2012. Would an anatomically shaped lumbar interbody cage provide better stability? An in vitro cadaveric biomechanical evaluation. Journal of spinal disorders \& techniques. 25(8):E240-244.

Wagnac E, Arnoux PJ, Garo A, Aubin CE. 2012. Finite element analysis of the influence of loading rate on a model of the full lumbar spine under dynamic loading conditions. Medical \& biological engineering \& computing. 50(9):903915.

Xu H, Ju W, Xu N, Zhang X, Zhu X, Zhu L, Qian X, Wen F, Wu W, Jiang F. 2013. Biomechanical comparison of transforaminal lumbar interbody fusion with 1 or 2 cages by finite-element analysis. Neurosurgery. 73(2):198-205. 


\section{$1 \quad$ List of figures}

2 Figure 1 a) The un-instrumented FE model of the L4-L5 segment including the vertebrae,

3 seven spinal ligaments, and intervertebral disc; b) regional thickness of the cortical bone

4 and finer mesh of the trabecular bone around the screw imprint ALL: Anterior

5 Longitudinal ligament, PLL: Posterior Longitudinal Ligament, ITL: Intertransverse

6 Ligament, CL: Capsular Ligament, LF: Ligament Flavum, ISL: Interspinous Ligament,

7 SSL: Supraspinous Ligament, AF: Annulus Fibrosus, NP: Nucleus Pulposus.

9 Figure 2 Simulation of different surgical procedures of TLIF: (a) Partial discectomy and 10 facetectomy of L4-L5, (b) Cage placement by imposing distractive force and moment on 11 L4, while the inferior endplate of L5 was fixed in space, and (c) Implementation of the 12 posterior fixation followed by application of the follower-load and physiological 13 moments (flexion, extension, lateral bending, and torsion) on the superior endplate of L4 14 while the inferior endplate of L5 was fixed in space.

15 Figure 3 Simulated placement scenarios of the cage: (a) Oblique asymmetric: (b) 16 Anterior symmetric.

17 Figure 4 Simulated ROM of the FEM of L4-L5 segment was within the reported range 18 of similar experimental tests on human cadaveric spines (Dahl et al. 2013; Jaramillo et al. 19 2016).

20 Figure 5 Range of motion (ROM) of the instrumented spinal segment in different 21 loading directions for normal (a) and osteoporotic (b) bone models (A08/A10: Oblique asymmetric placement of 8/10-mm cage; S08/S10: Anterior symmetric placement of 8/10-mm cage). 
1 Figure 6 Maximum Von-Mises stress at the endplate-cage interface in different loading

2 directions for normal (a) and osteoporotic (b) bone model (A08/A10: Oblique asymmetric

3 placement of 8/10-mm cage; S08/S10: Anterior symmetric placement of 8/10-mm cage).

4 Figure 7 Stress (in MPa) on the superior endplate of L5 under simulated flexion

5 moment of $10 \mathrm{~N} \mathrm{~m}$ and $400 \mathrm{~N}$ follower-load.

6 Figure 8 Maximum Von-Mises stress in the posterior rods in different loading directions

7 for normal (a) and osteoporotic (b) bone models (A08/A10: Oblique asymmetric

8 placement of 8/10-mm cage; S08/S10: Anterior symmetric placement of 8/10-mm cage)

9

10 